COMUNICACIÓN BREVE

\title{
COVID-19 en terapia intensiva: ¿qué pacientes tienen más riesgo de requerir ventilación mecánica?
}

\author{
Recibido: 8/11/20 Aceptado: 20/12/20
}

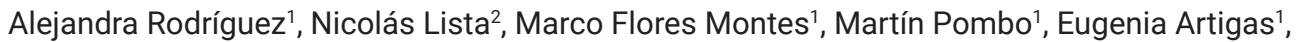
Ignacio Prieto ${ }^{1}$, Mirta Peralta ${ }^{1}$, María Inés Calderón ${ }^{1}$, Lautaro de Vedia ${ }^{3}$, Eleonora Cunto ${ }^{4}$.

\section{RESUMEN}

Introducción: En COVID-19 grave, identificar pacientes con mayor riesgo de ventilación mecánica (VM) ayuda a optimizar el manejo.

Materiales y métodos: Análisis retrospectivo de características clínico-epidemiológicas de pacientes con COVID-19 ingresados a UTI entre marzo/septiembre 2020. Se utilizó estadística descriptiva, análisis univariado ( $p$ significativa $<0.05$ ) y multivariado (variables significativas en el univariado).

Resultados: Ingresaron 114 pacientes, 91 (79,8\%) hombres, edad promedio $54,6 \pm 14,8$ años. 66 (57,9\%) eran obesos, $44(38,6 \%) \geq 60$ años, $38(33,3 \%)$ hipertensos $(33,3 \%)$ y $28(24,6 \%)$ diabéticos. 39 (23\%) tenían ferritina elevada, $62(76,5 \%)$ linfopenia y $33(41,3 \%)$ LDH aumentada. Mortalidad global $34 \%$. 72 pacientes $(63,1 \%)$ requirieron VM. Los predictores independientes de requerimiento de $\mathrm{VM}$ en el análisis multivariado fueron sexo masculino, edad $\geq 60$ años, diabetes, linfopenia y ferritina y LDH aumentadas.

Conclusiones: En pacientes con COVID-19 grave, los predictores independientes de necesidad de VM fueron sexo masculino, edad $\geq 60$ años, diabetes, niveles elevados de ferritina y LDH y linfopenia.

Palabras clave: COVID-19, SARS-CoV-2, ventilación mecánica, terapia intensiva.

\author{
${ }^{1}$ Unidad Asistencia Respiratoria, Hospital Francisco J. \\ Muñiz, CABA, Argentina. \\ ${ }^{2}$ Unidad Terapia Intermedia, Hospital Francisco J. Muñiz, \\ CABA, Argentina. \\ ${ }^{3}$ División Asistencia Especial, Departamento de Asistencia \\ Intensiva al Paciente Infectológico Crítico, Hospital \\ Francisco J. Muñiz, CABA, Argentina \\ ${ }^{4}$ Departamento de Asistencia Intensiva al Paciente \\ Infectológico Crítico, Hospital Francisco J. Muñiz, CABA, \\ Argentina
}

Autor para correspondencia: Dra. Alejandra Rodríguez, Marcelo T. de Alvear 19348 "A", CP (1122), CABA, Argentina.

Email: alejandrarodriguez28967@gmail.com

Este trabajo no cuenta con financiamiento. Ninguno de los autores presenta conflicto de intereses con relación a esta publicación. 


\section{Introducción}

La infección por SARS-CoV-2 puede provocar la enfermedad por coronavirus 19 (COVID-19) (1, 2). Si bien la mayoría de los pacientes son asintomáticos o cursan enfermedad leve, aproximadamente el $14 \%$ desarrolla un cuadro más grave, con requerimiento de internación en unidades de terapia intensiva (UTI) y potencial necesidad de ventilación mecánica (VM) (3).

Uno de los principales desafíos es cómo identificar rápidamente a los pacientes con alto riesgo de peores resultados. Sin embargo, los indicadores de alerta temprana eficaces todavía son limitados (4). La identificación de predictores tempranos de la necesidad de $\mathrm{VM}$ al ingreso de los pacientes adquiere una importancia crítica, ya que podría ayudar a mejorar el pronóstico (5).

El objetivo de este trabajo es presentar las características de los pacientes con COVID-19 que ingresaron a UTI y evaluar cuáles fueron los factores de riesgo asociados con necesidad de VM.

\section{Materiales y métodos}

Se incluyeron los pacientes con diagnóstico confirmado de COVID-19 que ingresaron en una UTI de un hospital público de la Ciudad Autónoma de Buenos Aires (CABA) entre marzo y septiembre de 2020. Se analizaron retrospectivamente características epidemiológicas, clínicas y de laboratorio al ingreso, así como diferentes factores de riesgo potencialmente asociados con necesidad de VM. Se utilizó estadística descriptiva, y para explorar los factores asociados con mortalidad, análisis univariado con test exacto de Fisher para variables categóricas y t-test para variables continuas. Una $p \leq 0.05$ fue considerada significativa. Se realizó análisis multivariado con regresión logística para aquellas variables que exhibieron un valor de $p$ significativo en el análisis univariado.

\section{Resultados}

Se evaluaron 114 pacientes con COVID-19, de los cuales $91(79,8 \%)$ eran de sexo masculino. El promedio de edad fue de $54,6 \pm 14,8$ años. El 38,6\% (44 pacientes) era mayor de 60 años. El promedio de días transcurridos entre la fecha de inicio de síntomas (FIS) y el ingreso a UTI fue de 7 días. Entre los factores de riesgo presentes se observó obesidad (76 pacientes; 57,9\%), hipertensión arterial (38; $33,3 \%)$, diabetes $(28 ; 24,6 \%)$, asma bronquial $(10 ; 8 \%)$, $\operatorname{EPOC}(7 ; 6,1 \%), \operatorname{HIV}(6 ; 5 \%)$ e insuficiencia renal crónica
(3; $2,5 \%$ ). El valor promedio de ferritina fue de 1490,2 $\pm 937,1 \mathrm{mcg} / \mathrm{L}$, y un $34 \%$ de los casos (39 pacientes) tuvo un valor mayor a $1000 \mathrm{mcg} / \mathrm{L}$. El valor promedio de Dímero-D alcanzó 1280,9 \pm 1010,7 ng/mL, y 38,5\% de los casos (44 pacientes) presentaron valores superiores a $500 \mathrm{ng} / \mathrm{mL}$. El valor promedio de linfocitos fue de 1080,7 $\pm 707,5 / \mathrm{mm}^{3}$, y un $76,5 \%$ de los pacientes mostró valores inferiores a $1000 / \mathrm{mm}^{3}$. El valor promedio de LDH fue de $694 \pm 255,6 \mathrm{UI} / \mathrm{L}$, con un $41,3 \%$ de los pacientes con valores superiores a $700 \mathrm{UI} / \mathrm{L}$. La mortalidad global fue de 34\% (39 pacientes). Las características demográficas, antecedentes patológicos y parámetros de laboratorio al ingreso se presentan en la Tabla 1.

De los 114 pacientes analizados, $72(63,1 \%)$ requirieron VM, mientras que en los 42 restantes (36,9\%) el abordaje pudo realizarse sin necesidad de respirador (oxigenoterapia

\section{Tabla 1. Características demográficas, clínicas y de laboratorio de los pacientes incluidos}

\begin{tabular}{|c|c|}
\hline & $\begin{array}{c}\text { Total } \\
(n=114)\end{array}$ \\
\hline Sexo, n (\%) & $91(79,8 \%)$ \\
\hline Edad promedio (años \pm DE) & $54,6 \pm 14,8$ \\
\hline Edad >60 años, n (\%) & $44(38,6 \%)$ \\
\hline Días FIS/Ingreso a UTI (mediana) & 7 \\
\hline HIV, n (\%) & $6(5 \%)$ \\
\hline Diabetes, n (\%) & $28(24,6 \%)$ \\
\hline Hipertensión arterial, n (\%) & $38(33,3 \%)$ \\
\hline IRC, n (\%) & $3(2,5 \%)$ \\
\hline EPOC, n (\%) & $7(6,1 \%)$ \\
\hline Asma, n (\%) & $10(8,8 \%)$ \\
\hline IMC>30, n (\%) & $76(57,9 \%)$ \\
\hline Ferritina (mcg/L, promedio) & $1490,2 \pm 937,1$ \\
\hline Ferritina>1000 mcg/L, n (\%) & $39(34 \%)$ \\
\hline Dímero D (ng/mL, promedio) & $1280,9 \pm 1010,7$ \\
\hline Dímero D >500ng/mL, n (\%) & $44(38,5 \%)$ \\
\hline Linfocitos (/mm³ ${ }^{3}$ promedio) & $1080,7 \pm 707,5$ \\
\hline Linfocitos<1000/mm³, n (\%) & $52(76,5 \%)$ \\
\hline LDH (UI/L, promedio) & $694 \pm 255,6$ \\
\hline LDH > 700/UI/L, n (\%) & $33(41,3 \%)$ \\
\hline Mortalidad, n (\%) & $39(34 \%)$ \\
\hline
\end{tabular}

Abreviaturas: FIS: fecha de inicio de síntomas; IRC: insuficiencia renal crónica; IMC: índice de masa corporal; EPOC: enfermedad pulmonar obstructiva crónica; LDH: láctico dehidrogenasa 
Tabla 2. Comparación de las características demográficas, clínicas y de laboratorio entre los dos subgrupos de pacientes (VM y noVM)

\begin{tabular}{|c|c|c|c|}
\hline & $\begin{array}{c}\text { VM } \\
(n=72)\end{array}$ & $\begin{array}{l}\text { No VM } \\
(n=42)\end{array}$ & $\begin{array}{l}\text { Comparación VM vs } \\
\text { No VM, Valor de } p\end{array}$ \\
\hline Sexo, n (\%) & $62(86,1 \%)$ & $29(69 \%)$ & 0,02 \\
\hline Edad promedio (años $\pm D E$ ) & $59,9 \pm 13,3$ & $48,2 \pm 15,6$ & $<0,001$ \\
\hline Edad >60 años, $n(\%)$ & $34(47,2 \%)$ & $10(23,8 \%)$ & 0,01 \\
\hline Días FIS/Ingreso a UTI (mediana) & 7 & 7,5 & NS \\
\hline HIV, n (\%) & $5(6,7 \%)$ & $1(2,4 \%)$ & 0,57 \\
\hline Diabetes, n (\%) & $23(30,7 \%)$ & $5(11,9 \%)$ & 0,04 \\
\hline Hipertensión arterial, n (\%) & $26(34,7 \%)$ & $12(28,6 \%)$ & 0,6 \\
\hline IRC, n (\%) & $3(4 \%)$ & $0(0 \%)$ & NS \\
\hline EPOC, n (\%) & $5(6,7 \%)$ & $2(4,8 \%)$ & 0,99 \\
\hline Asma, n (\%) & $6(8 \%)$ & $4(9,5 \%)$ & NS \\
\hline $\mathrm{IMC}>30, \mathrm{n}(\%)$ & $43(57,3 \%)$ & $23(54,8 \%)$ & 0,94 \\
\hline Ferritina (mcg/L, promedio) & $1686,7 \pm 973$ & $962 \pm 580,1$ & 0,007 \\
\hline Ferritina>1000 mcg/L, n (\%) & $33(76,7 \%)$ & $6(37,5 \%)$ & $<0,001$ \\
\hline Dímero D (ng/mL, promedio) & $1378,4 \pm 1071,3$ & $1112,5 \pm 861,1$ & 0,36 \\
\hline Dímero D >500 ng/mL, n (\%) & $32(44,4 \%)$ & $12(28,5 \%)$ & 0,06 \\
\hline Linfocitos (/mm³, promedio) & $902,7 \pm 463,6$ & $1383,3 \pm 97,4$ & 0,002 \\
\hline Linfocitos $<1000 / \mathrm{mm}^{3}, \mathrm{n}(\%)$ & $39(76,5 \%)$ & $13(43,3 \%)^{\star \star}$ & $<0,001$ \\
\hline LDH (UI/L, promedio) & $743,1 \pm 245,8$ & $575,7 \pm 194$ & 0,002 \\
\hline LDH >700 UI/L, n (\%) & $27(51,9 \%)$ & $6(21,4 \%)$ & $<0,001$ \\
\hline Mortalidad, n (\%) & $39(54,2 \%)$ & $0(0 \%)$ & $<0,001$ \\
\hline
\end{tabular}

Abreviaturas: FIS: fecha de inicio de síntomas; IRC: insuficiencia renal crónica; IMC: índice de masa corporal; EPOC: enfermedad pulmonar obstructiva crónica; LDH: láctico dehidrogenasa

con máscara de reservorio). En la Tabla 2 se exhiben las características demográficas, antecedentes patológicos y parámetros de laboratorio de los dos subgrupos de pacientes (VM y noVM), con la comparación entre ambos mediante el análisis univariado. Allí puede observarse que los factores que presentaron diferencias significativas fueron el sexo masculino $(86,1 \%$ en el grupo de VM vs $69,1 \%$ en el grupo noVM, $p=0,02)$, la edad promedio $(59,9$ $\pm 13,3$ vs $48,2 \pm 15,6, p<0,001$ ), la edad mayor a 60 años ( $47,2 \%$ vs $23,8 \%$ ), el antecedente de diabetes $(30,7 \%$ vs $11,9 \%, p=0,04)$, el valor de ferritina mayor a $1000 \mathrm{mcg} / \mathrm{L}$ $(76,7 \%$ vs $37,5 \%, p<0,001)$, la linfopenia $(76,5 \%$ vs $43,3 \%$, $\mathrm{p}<0,001)$ y la LDH superior a $700 \mathrm{UI} / \mathrm{L}(51,9 \%$ vs $21,4 \%$, $p<0,001)$.

En la Tabla 3 y la Figura 1 se exhibe el análisis multivariado, en el cual se analizaron los factores de riesgo que se asociaron con requerimiento de VM. Los predictores independientes de requerimiento de VM en la población estudiada fueron el sexo masculino, la edad $\geq 60$ años, la diabetes, la linfopenia y el aumento de ferritina y LDH.

\section{Discusión}

La tasa de mortalidad informada de los pacientes con COVID-19 con VM varía del 30 al 97\% (6-8). Si bien los parámetros que definirán finalmente la conducta

Tabla 3. Análisis multivariado, predictores independientes asociados con requerimiento de VM

\begin{tabular}{|l|c|c|}
\multicolumn{1}{|c|}{ Factor } & OR & IC 95\% \\
\hline Ferritina $>1000 \mathrm{mcg} / \mathrm{L}$ & 5,50 & $1,60-18,91$ \\
\hline Linfocitos $<1000 \mathrm{~mm}^{3}$ & 4,25 & $1,61-11,21$ \\
\hline LDH $>700 \mathrm{UI} / \mathrm{L}$ & 3,96 & $1,38-11,36$ \\
\hline Diabetes & 3,47 & $1,21-10,0$ \\
\hline Edad $\geq 60$ años & 2,86 & $1,23-6,68$ \\
\hline Sexo masculino & 2,78 & $1,09-7,08$
\end{tabular}


Figura 1. Análisis multivariado, predictores independientes asociados con requerimiento de VM. Forest Plot.

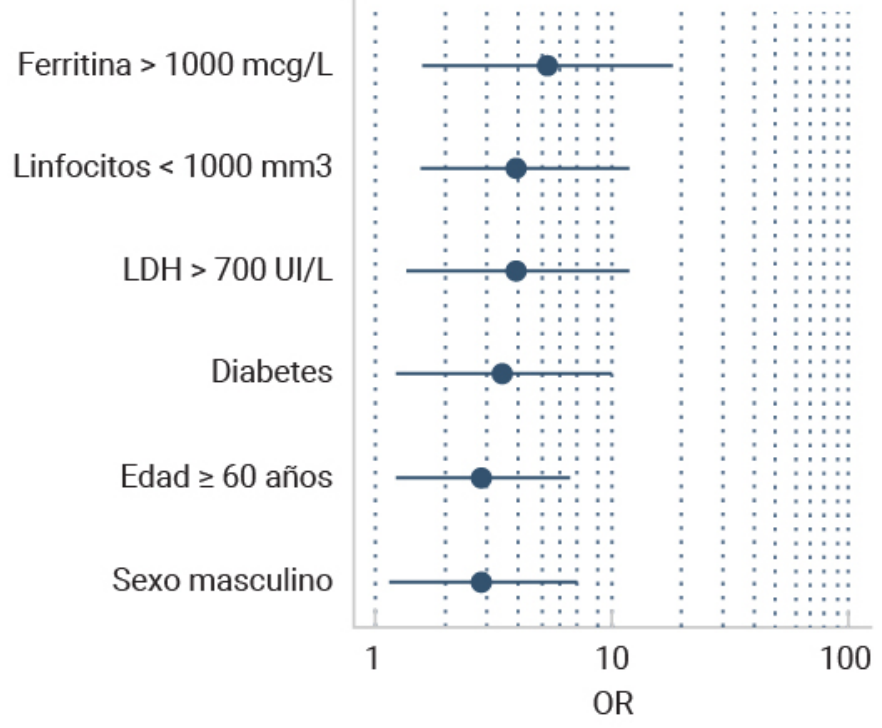

médica son principalmente respiratorios, gasométricos o hemodinámicos, entre otros, es relevante tratar de identificar los factores de riesgo asociados con la necesidad de VM a fin de optimizar el manejo de la oxigenoterapia o bien indicar VM de manera precoz (9). Se dispone de poca información sobre los factores de riesgo que pueden contribuir a incrementar la necesidad de VM en aquellos pacientes con formas graves de COVID-19 (10).

De acuerdo con informes anteriores, se observó que la edad avanzada es el predictor más significativo de resultados graves (11). Un estudio reciente en el que participaron 72.314 pacientes en China reveló que la tasa de letalidad fue del $8 \%$ para los individuos de 70 a 79 años y del $14,8 \%$ para los mayores de 80 (3). Otros reportes mostraron que el sexo masculino constituye un factor de riesgo de resultados desfavorables en pacientes con COVID-19 (12). El sexo puede influir en la gravedad del SARS-CoV-2, ya que el cromosoma $\mathrm{X}$ contiene una mayor densidad de genes relacionados con la inmunidad y elementos reguladores que se refieren a la inmunidad inherente y adaptativa, lo cual puede ser una de las posibles razones por las que las mujeres parecían ser menos susceptibles a la infección viral o la infección era más leve que en los hombres $(13,14)$. Una amplia revisión y metanálisis mostró que dos tercios de los pacientes con COVID-19 grave eran hombres, y con una edad promedio de 62,6 años (15). Estas dos características, la edad mayor a 60 años y el sexo masculino, fueron también factores de riesgo asociados con necesidad de VM en nuestra población.
Muchos estudios observaron que los pacientes con obesidad (IMC $>30 \mathrm{~kg} / \mathrm{m}^{2}$ ) tenían más probabilidades de cursar cuadros graves $(15,16)$. Sin embargo, en nuestra población, si bien más de la mitad de los pacientes eran obesos, esta condición no constituyó un factor independiente predictor de necesidad de VM. Lo mismo sucedió con la hipertensión arterial, que se observó en un tercio de la población estudiada. Por el contrario, la diabetes, presente en un cuarto de la población estudiada por nuestro grupo, sí constituyó un predictor independiente de necesidad de VM, tal como fue descripto en otros trabajos (15).

Varios parámetros de laboratorio han sido utilizados para predecir la evolución de los pacientes con infecciones graves por SARS-CoV-2 (17). El que mostró la mayor asociación con necesidad de VM fue el elevado nivel de ferritina en sangre (18). La ferritina en circulación aumenta durante las infecciones virales y puede ser un marcador de la replicación viral $(19,20)$. También se han informado niveles elevados de ferritina debido a la tormenta de citoquinas desencadenada en la infección grave por SARS-CoV-2 $(21,22)$. Sin embargo, la prueba de ferritina no siempre está disponible en una emergencia. En nuestro estudio, los pacientes con valores de ferritina mayores a $1000 \mathrm{mcg} / \mathrm{L}$ tuvieron más de cinco veces mayor riesgo de requerir VM.

La linfopenia se observa a menudo en pacientes con COVID-19, especialmente en los graves que ingresan a UTI $(4,23,24)$. En nuestra población también observamos que los sujetos con menor cantidad de linfocitos tuvieron más posibilidades de evolucionar con insuficiencia respiratoria grave y necesidad de VM.

Por último, la LDH es una enzima glucolítica citoplasmática que se expresa en todos los tejidos y su elevación sugiere daño tisular, especialmente daño hepático y cardíaco. Se observó un aumento de LDH en pacientes con infección por SARS-CoV-2 (25) y se predijo como un indicador de gravedad y pronóstico en COVID-19 (26). En nuestro estudio, la LDH elevada fue un predictor eficaz de necesidad de VM entre los pacientes con COVID-19.

En las series principales de pacientes con COVID-19 publicadas hasta la fecha, los pacientes con peor pronóstico han mostrado niveles de D-dímero mucho más altos que los individuos con enfermedad menos grave (27-30). Sin embargo, en nuestra población este parámetro fue muy elevado en ambos grupos, tanto 
en aquellos que requirieron VM como en los que se pudieron manejar con oxígenoterapia con máscara de reservorio, por lo que no obró como factor predictor de necesidad de VM. Posiblemente, la cantidad de pacientes no fue lo suficientemente grande como para poder evidenciar diferencias significativas desde el punto de vista estadístico. No fueron considerados los valores de procalcitonina y de PCR, dado que dichas determinaciones se realizaron en una porción minoritaria del grupo de pacientes estudiados.

Este estudio tiene algunas debilidades: fue realizado en un solo centro y el tamaño de la muestra no es tan grande, lo que impidió una evaluación más sólida de los factores de riesgo asociados. Finalmente, dada su naturaleza retrospectiva, tampoco podemos asegurar que ninguna de nuestras intervenciones afectó directamente los resultados de los pacientes.

En conclusión, de acuerdo con los datos observados en nuestra población, los factores que se asociaron con requerimiento de VM en pacientes con COVID-19 grave fueron sexo masculino, edad mayor a 60 años, diabetes, niveles elevados de ferritina y LDH, y linfopenia. 


\section{Bibliografía}

1. Lu R., Zhao X., Li J., Niu P., Yang B., Wu H. Genomic characterisation and epidemiology of 2019 novel coronavirus: implications for virus origins and receptor binding. Lancet. 2020; 395 (10224): 565-74.

2. Zhu N., Zhang D., Wang W., Li X., Yang B., Song J. A novel coronavirus from patients with pneumonia in China, 2019. N Engl J Med. 2020; 382 (8): 727-33.

3. Wu Z., McGoogan J.M. Characteristics of and important lessons from the coronavirus disease 2019 (COVID-19) outbreak in China: summary of a report of 72314 cases from the Chinese Center for Disease Control and Prevention. JAMA. 2020; 323: 1239-42.

4. LeiC,LinW,DengX,HuF,ChenF,CaiW,etal. Factorsassociated with clinical outcomes in patients with Coronavirus Disease 2019 in Guangzhou, China. J Clin Virol. 2020 Oct 14; 133: 104661.

5. Hu H, Du H, Li J, Wang Y, Wu X, Wang C, et al. Early prediction and identification for severe patients during the pandemic of COVID-19: A severe COVID-19 risk model constructed by multivariate logistic regression analysis. Glob Health. 2020 Dec; 10 (2): 020510.

6. Richardson S, Hirsch JS, Narasimhan M, Crawford JM, McGinn T, Davidson KW, et al. Presenting characteristics, comorbidities, and outcomes among 5700 patients hospitalized with COVID-19 in the new York City area. JAMA. 2020;10022:E1-E8.

7. Auld SC, Caridi-Scheible M, Blum JM, Robichaux C, Kraft C, Jacob JT, et al. ICU and ventilator mortality among critically ill adults with coronavirus disease 2019. Crit Care Med. 2020;48:e799-e804.

8. Quah P,Li A, Phua J. Mortality rates of patients with COVID-19 in the intensive care unit: a systematic review of the emerging literature. Crit. Care. NLM (Medline) 2020; 24: 285.

9. David P, Garcia W, Fumeaux T, Guerci P, Monika D, Montomoli $J$, et al. Prognostic factors associated with mortality risk and disease progression in 639 critically ill patients with COVID-19 in Europe: Initial report of the international RISC-19-ICU prospective observational cohort. EClinicalMedicine. 2020; 25: 100449.

10. Yang X, Yu Y, Xu J, Shu H, Xia J, Liu H, et al. Clinical course and outcomes of critically ill patients with SARS-CoV-2 pneumonia in Wuhan, China: a single-centered, retrospective, observational study. Lancet Respir Med. 2020; 8: 475-81.

11. Du R.-H., Liang L.-R., Yang C.-Q., Wang W., Cao T.-Z., Li M. Predictors of mortality for patients with COVID-19 pneumonia caused by SARS-CoV-2: a prospective cohort study. Eur Respir J. 2020; 55 (5): 2000524.

12. Gupta S., Hayek S.S., Wang W., Chan L., Mathews K.S.,
Melamed M.L. Factors associated with death in critically ill patients with coronavirus disease 2019 in the US. JAMA Intern Med. 2020;15 doi:10.1001/jamainternmed.2020.3596. Published online July.

13. Schurz, H, Salie, M, Tromp, G, et al. The X chromosome and sex-specific effects in infectious disease susceptibility. Hum Genomics 2019; 13: 2.

14. Vemuri, R, Sylvia, KE, Klein, SL, et al. The microgenderome revealed: sex differences in bidirectional interactions between the microbiota, hormones, immunity and disease susceptibility. Semin Immunopathol 2019; 41: 265-275.

15. Tan E, Song J, Deane AM, Plummer MP. Global impact of COVID-19 infection requiring admission to the intensive care unit: a systematic review and meta-analysis. Chest. 2020 Oct. 15: S0012-3692(20)34906-0.

16. Kim T, Roslin M, Wang JJ, Kane J, Hirsch JS, Ji Kim E; Northwell. Body Mass Index as a Risk Factor for Clinical Outcomes in Patients Hospitalized with COVID-19 in New York. Health COVID-19 Research Consortium.Obesity (Silver Spring). 2020 Oct 31. doi: 10.1002/oby.23076.

17. An,PJ,Zhu, YZ, Yang, LP.Biochemical indicators of coronavirus disease 2019 exacerbation and the clinical implications. Pharmacol Res 2020; 159: 104946.

18. Chen L, Li H, Li L, Liu C, Yan S, Chen H, Li Y. Ferritin in the coronavirus disease 2019 (COVID-19): A systematic review and meta-analysis. .J Clin Lab Anal. 2020 Oct 19; e23618. Doi: 10-1002/jcla23618

19. Li Y, Hu Y, Yu J, Ma T. Retrospective analysis of laboratory testing in 54 patients with severe- or critical-type 2019 novel coronavirus pneumonia. Lab Invest. 2020 Jun; 100 (6): 794800.

20. Baraboutis IG, Gargalianos P, Aggelonidou E, Adraktas A; Collaborators.Initial Real-Life Experience from a Designated COVID-19 Centre in Athens, Greece: a Proposed Therapeutic Algorithm. SN Compr Clin Med. 2020 May 26: 1-5.

21. Velavan TP, Meyer CGZ. Mild versus severe COVID-19: Laboratory markers..Int J Infect Dis. 2020 Jun; 95: 304-7.

22. Giamarellos-Bourboulis EJ, Netea MG, Rovina N, Akinosoglou K, Antoniadou A, Antonakos N, et al. Complex Immnue Dysregulation in COVID-19 Patients with Severe Respiratory Failure. Cell Host Microbe. 2020 Jun 10; 27 (6): 992-1000.e3.

23. Cao X. COVID-19: immunopathology and its implications for therapy. Nat Rev Immunol. 2020 May; 20 (5): 269-70.

24. Liu Z, Long W, Tu M, Chen S, Huang Y, Wang S, et al. Lymphocyte subset (CD4+, CD8+) counts reflect the severity of infection and predict the clinical outcomes in patients with COVID-19. J Infect. 2020 Aug; 81 (2): 318-56.

25. Booth, CM, Matukas, LM, Tomlinson, GA, Rachlis AR, RoseDB, Qwosh HA, et al. Clinical features and short-term outcomes of 144 patients with SARS in the greater Toronto area. JAMA. 
2003; 289: 2801-9.

26. Poggiali, E, Zaino, D, Immovilli, P, et al. Lactate dehydrogenase and $\mathrm{C}$-reactive protein as predictors of respiratory failure in CoVID-19 patients. Clin Chim Acta. 2020; 509: 135-8.

27. Lippi G, Favaloro EJ. D-dimer is Associated with Severity of Coronavirus Disease 2019: A Pooled Analysis. Thromb Haemost. 2020 Apr 3. doi: 10.1055/s-0040-1709650.

28. Huang C, Wang Y, Li X, Ren L, Zhao J, Hu Y, et al. Clinical features of patients infected with 2019 novel coronavirus in Wuhan, China. Lancet. 2020 Feb 15; 395 (10223): 497-506.

29. TangN,LiD, Wang X,SunZ. Abnormal coagulation parameters are associated with poor prognosis in patients with novel coronavirus pneumonia. J Thromb Haemost. 2020 Apr, 18 (4): 844-7.

30. Zhou F, Yu T, Du R, Fan G, Liu Y, Liu Z, et al. Clinical course and risk factors for mortality of adult inpatients with COVID-19 in Wuhan, China: a retrospective cohort study. Lancet. 2020 Mar 28;395(10229):1054-1062. 
COVID-19 in intensive care: which patients are most at risk of requiring mechanical ventilation?

Introduction: In severe COVID-19, identifying patients with a higher risk of mechanical ventilation (MV) helps to optimize management.

Materials and methods: Retrospective analysis of clinicalepidemiological characteristics of patients with COVID-19 admitted to ICU between March / September 2020. Descriptive statistics, univariate and multivariate analysis were used.

Results: 114 patients were admitted, 91 (79.8\%) were men, mean age $54.6 \pm 14.8$ years. 66 (57.9\%) were obese, 44 $(38.6 \%) \geq 60$ years, 38 (33.3\%) were hypertensive (33.3\%) and $28(24.6 \%)$ diabetics. 39 (23\%) had elevated ferritin, 62 (76.5\%) had lymphopenia, and $33(41.3 \%)$ had increased LDH. Overall mortality $34 \% .72$ patients $(63.1 \%)$ required MV. The independent predictors of $\mathrm{MV}$ requirement in the multivariate analysis were male sex, age $\geq 60$ years, diabetes, lymphopenia, and increased ferritin and LDH.

Conclusions: In patients with severe COVID-19, the independent predictors of the need for MV were male sex, age $\geq 60$ years, diabetes, elevated levels of ferritin and LDH, and lymphopenia.

Keywords: COVID-19, SARS-CoV-2, mechanical ventilation, intensive care. 